\title{
Integrated Opto-Mechanical Modeling with Scene-Based Metrology Techniques for Structural Dynamics Compensation
}

\author{
Eugene Fahnestock ${ }^{1}$, Robert Fuentes ${ }^{2}$, R. Scott Erwin ${ }^{3}$
}

\begin{abstract}
This paper provides a summary of recent work in the development of integrated, multi-physics models for controlled opto-mechanical systems. Standard approaches from the literature are used to model the dynamics of the structure, including piezoceramic actuators, and generate a simple state space system for the actuator-structure dynamics. Next, linear sensitivities of the coefficients of the standard Zernike orthonormal basis set for representing optical aberrations are generated with respect to motions of the optics around the equilibrium point, using commercially available ray-trace software. Using this linear sensitivity representation, the optical path difference (OPD) at a reference pupil plane is reconstructed. Inclusion of additional defocus terms to these Zernike coefficients yields a second aberration function to allow for the reconstruction of the OPD at a defocused pupil plane as well. For both of these planes, Fourier analysis is used to obtain the images produced at the corresponding image plane. The two images are provided to a phase diversity algorithm that returns estimates of the Zernike coefficients, yielding an estimated aberration based on sensed images. Laser metrology sensor signals are modeled via the addition of physically realistic noise to the structural perturbation signals, and are used to develop a control system that autoaligns the structure based on both the laser and phase diversity sensor measurements. The processes described herein are demonstrated on a model of an opto-mechanical system based on an approximate prescription for the Hubble Space Telescope combined with a simple flexible structure.
\end{abstract}

\section{INTRODUCTION}

While the two fields of structural modeling and optics are each themselves well established, the intersection of those areas with each other and the use of control systems that utilize information from both for achieving optical performance objectives directly is a relatively new topic of research. State-space modeling of structures has been well documented, with the option of incorporating actuators (see [1], [2]). Software packages such as CODE V, ZEMAX, MACOS, and COMP exist to model optical systems via raytracing, with the latter two providing optics models more easily integrated with structural and thermal models through the optional computation of linear sensitivities (see [3], [4]). Some software packages such as IMOS [5] have been developed to actually accomplish this integration. Integrated modeling of structural, thermal, and optical performance has been carried out for the Next-Generation Space Telescope

\footnotetext{
${ }^{1}$ Student, Department of Aerospace Engineering and Aviation, The Ohio State University, Columbus, OH 43210-1278, fahnestock.2@osu.edu

${ }^{2}$ Boeing-SVS Inc., 4411 The 25 Way NE, Suite 350, Albuquerque, NM $87109-5858$

${ }^{3}$ Air Force Research Laboratory, Space Vehicles Directorate, 3550 Aberdeen Ave. SE, Kirtland AFB, NM 87117-5776
}

(NGST) using ray-tracing [6], and using linear sensitivities rather than ray-tracing with the addition of feedback control [7]. In this paper, we will also investigate the impact of using image-based metric determination (i.e., phase diversity methods), as described in [8], [9], and [10], with the structural dynamics feedback control system. Specifically, we will incorporate compensation for non-common path errors between metrology and imaging optical paths while simultaneously compensating for structural vibration. The processes described herein are demonstrated on a model of an opto-mechanical system based on an approximate prescription for the Hubble Space Telescope combined with a simple flexible structure (i.e. the example system).

\section{ACTUATOR-STRUCTURE MODELING}

For the example system, the optical prescription is modeled using the ZEMAX optical design program. The prescription consists of a Schmidt-Cassegrain telescope with an obscuration to prevent rays from passing through the back side of the hyperbolic secondary mirror having diameter 0.281 meters. The parabolic primary mirror has a diameter of 2.4 meters and is the aperture stop for the system. The separation between the primary and secondary mirrors along the optical axis is approximately 4.906 meters.

The position data for the optical surfaces within this prescription was exported from ZEMAX as an IGES file and imported into FEMAP, in which the simple structural FE model was created to match the surfaces. Square shell elements were used for most of the primary and secondary mirror surfaces with triangular shell elements connecting at the central nodes on the optical axis completing those surfaces. A combination of square and triangular shell elements were used to form a triangular base plate representing a spacecraft bus. The mirrors and base plate formed by the shells were considered rigid for this entire integrated modeling process. The shells were connected by beam elements to form a tripod support connecting the primary and secondary mirrors and a hexapod support, into which the piezoelectric actuators were integrated, connecting the primary mirror and bus. The completed FE model, shown in Fig. 1, was exported as an .INP file. This was imported into ABAQUS standard, which was used to perform a Lanczos modal dynamic solve to obtain 32 non-rigid body modal frequencies up to approximately $200 \mathrm{~Hz}$ and the accompanying mode shape displacements at nodes of interest. These were the mirror vertex nodes and the nodes at the endpoints of the actuator elements. The displacements on the former nodes were assembled into a matrix $\varphi$ and those on the 


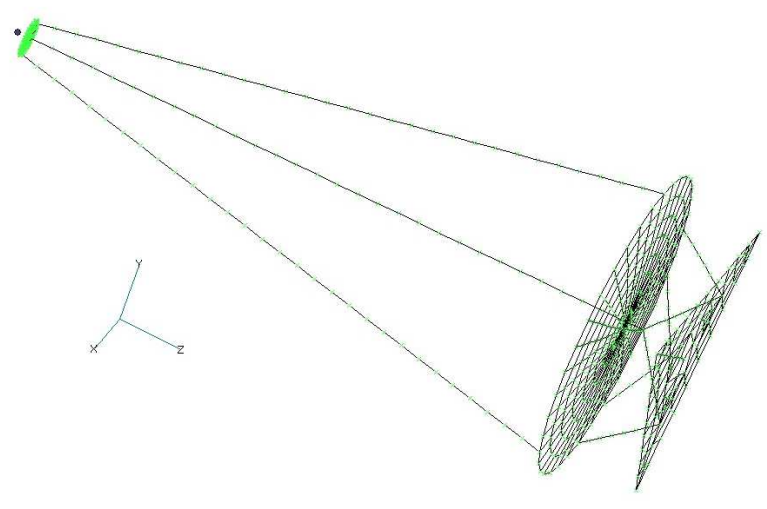

Fig. 1. Finite-Element Model of Example System

latter were assembled into a matrix $\tilde{\varphi}$. The blocked force acting per unit voltage on each piezoelectric actuator was calculated via standard techniques [11], and broken down into its vector components within the global coordinate system. The resolved vector components were assembled into a matrix $\hat{b}$. The continuous state-space system was formulated using standard approaches [2], yielding

$$
I \ddot{q}+[\Xi] \dot{q}+\left[\Omega^{2}\right] q=[\tilde{\varphi} \hat{b}] u,
$$

where

$$
\begin{array}{r}
\Xi=\operatorname{diag}\left(2 \zeta_{j} \omega_{j}\right), \\
\Omega^{2}=\operatorname{diag}\left(\omega_{j}^{2}\right) .
\end{array}
$$

Here $j$ varies along the diagonal from 1 up to the total number of modes retained from the normal modes analysis, and $q$ represents the modal coordinate state vector. Conversion of (1) into a set of first-order differential equations yields

$$
\begin{aligned}
& \dot{x}=A x+B u, \\
& y=C x+D u,
\end{aligned}
$$

where

$$
A=\left[\begin{array}{cc}
-\Xi & -\Omega^{2} \\
I & 0
\end{array}\right], B=\left[\begin{array}{c}
\tilde{\varphi} \tilde{b} \\
0
\end{array}\right], C=[0 \varphi], D=[0],
$$

and $x^{T}=\left[\begin{array}{ll}\dot{q}^{T} & q^{T}\end{array}\right]$. Damping factors of $\zeta=0.5 \%, i=$ $1,2, \ldots, n$ were assumed. The continuous state-space model was then converted to its discrete-time equivalent using zero-order hold equivalence for use in digital control system simulation within Simulink.

\section{GENERATION OF OPTICAL QUANTITIES OF INTEREST}

Optical quantities of interest, such as line-of-sight pointing error, spot centroids, wave-front error (WFE), and optical-path length difference (OPD) are, in general, nonlinear functions of the positions of the surfaces representing the various lenses, mirrors, and other light-impacting elements of the system. This is due to the influence of both the geometric relationships governing reflection and refraction, and diffraction effects. Diffraction effects become more pronounced relative to the geometric effects with increasing distance from the optical system's aperture, or equivalently with decreasing distance from the focal (image) plane of the system (see [12], [13]).

The OPD was the optical quantity of interest obtained at the start of the process outlined in the remainder of this section. For this paper, the OPD at any given location on the pupil plane is defined as the length of a ray hitting the pupil plane at that location minus the length of the chief (on-optical-axis) ray measured up to the pupil plane. The OPD data was obtained at the pupil plane, which is quite close to the image plane. This point is within the diffractiondominated (Fraunhofer) region, satisfying one condition for the validity of the Fourier analysis covered below.

\section{A. Geometric Effects and Derivation of Sensitivities}

Modeling of the nonlinearities due to geometry is generally performed using ray-trace algorithms, that geometrically trace bundles of light rays through the system, tracking the quantities of interest throughout. Elements can be moved, perturbed, and surfaces modified to ascertain the impact on metrics of interest. CODE V, ZEMAX, MACOS, and COMP all offer this ray-trace-based functionality.

Unfortunately, ray-tracing is a computationally intensive procedure, and would require an unacceptable amount of time for simulating the system if integrated directly. The use of linearized optical sensitivities has been proposed and utilized with great success in generating several metrics of interest (see [14] and [15]). We shall follow this approach in this paper, focusing on the use of sensitivities to map from structural deformation to global optical properties rather than from structural deformation to individual-ray properties. Image generation will also be performed drawing upon the output of this mapping.

For the derivation of the sensitivities matrix, a software package that integrates ZEMAX with the MATLAB/Simulink software, ZELINK, was used. ZELINK consists of several Simulink-compatible blocks that will load a ZEMAX prescription file and construct inputs related to the 6 mechanical degrees of freedom (DOFs) for each defined surface, with several standard optical output functions (OPD, X- and Y-centroids, etc.) defined as the outputs. Using ZELINK blocks to represent the optical system, a simple Simulink system was constructed to feed a ramp input signal into each mechanical DOF for the primary and secondary mirrors, generating OPD surfaces as a function of perturbation value.

In order to represent each OPD surface with high fidelity using linear approximation techniques, a standard orthonormal decomposition of the surface was performed and the sensitivity of the coefficients of the expansion determined for each mechanical DOF in the system. The Zernike standard polynomials [16] were calculated for coordinates interior to the aperture function on the pupil plane. Each 


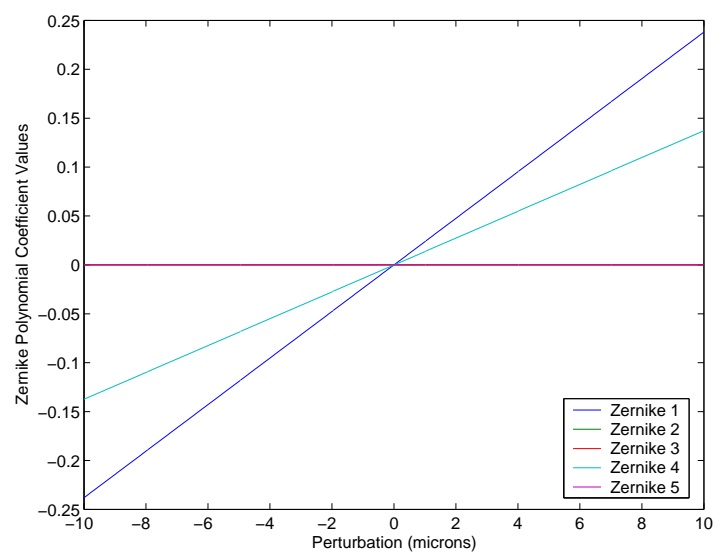

Fig. 2. Zernike Polynomial Coefficient Values vs. Primary Mirror Piston Perturbation

two-dimensional Zernike polynomial array matrix was reshaped into a vector and these were assembled into a matrix containing all the information of the orthonormal basis that is needed to perform the decomposition. Determination of the coefficients of the expansion for a given OPD surface was then accomplished using standard least-squares regression techniques. Using the OPD surfaces corresponding to a linearly increasing perturbation of a particular DOF (through zero), the coefficients for each basis function were each plotted against the input perturbation to examine the coefficient behavior. An example of such a plot is shown in Fig. 2, providing the behavior of the first 5 Zernike expansion coefficients with respect to the value of the perturbation in the piston (z-axis translation) DOF for the primary mirror.

Second-order accurate forward, central, and backward finite differencing was used to get the first derivatives of these curves, providing values for the sensitivity of each coefficient value to motion of the selected mechanical DOF. A plot of this first derivative for one Zernike coefficient is shown in Fig. 3. Clearly, numerical noise in the data is

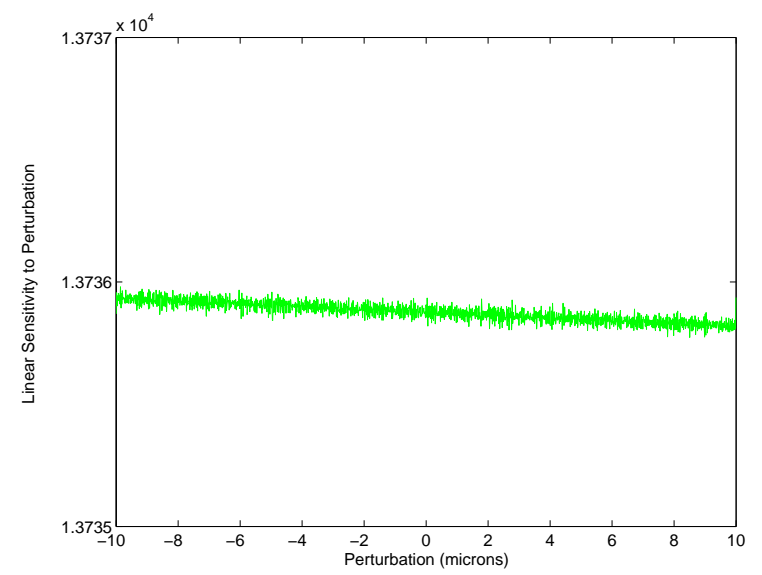

Fig. 3. Sensitivity of 4th Zernike Coefficient With Respect to Primary Mirror Piston DOF (unfiltered)
TABLE I

ERror IN OPD PREDICTION DUe to USE OF LiNEAR SENSITIVITIES

\begin{tabular}{|c|l|c|}
\hline \multicolumn{2}{|c|}{ Perturbation } & $\begin{array}{c}\text { Mean of Norm of Error } \\
\text { Vector Normalized by } \\
\text { Norm of Actual Vector }\end{array}$ \\
\hline \multirow{5}{*}{ Primary } & X-Decenter & 0.0008 \\
\cline { 2 - 3 } & Y-Decenter & 0.0008 \\
\cline { 2 - 3 } & Z-Piston & 0.0043 \\
\cline { 2 - 3 } & Tilt About X & 0.0009 \\
\cline { 2 - 3 } & Tilt About Y & 0.0009 \\
\cline { 2 - 3 } & Rotation About Z & 1.0090 \\
\hline \multirow{5}{*}{ Secondary } & X-Decenter & 0.0008 \\
\cline { 2 - 3 } & Y-Decenter & 0.0008 \\
\cline { 2 - 3 } & Z-Piston & 0.0043 \\
\cline { 2 - 3 } & Tilt About X & 0.0010 \\
\cline { 2 - 3 } & Tilt About Y & 0.0010 \\
\cline { 2 - 3 } & Rotation About Z & 1.0044 \\
\hline
\end{tabular}

amplified during the finite differentiation process. However, this effect was eliminated by taking the mean of the linear sensitivity over the entire prescribed perturbation range to get one number for the corresponding element of the linear sensitivities matrix. This perturbation range was chosen as $\pm 10 \mu \mathrm{m}$ or $\pm 10 \mu \mathrm{rad}$, representing values that were estimated to be approximately one order-of-magnitude larger than typically allowable vibration specifications for a spacebased imaging system.

The quality of the sensitivities matrix thus obtained was assessed by comparing the OPD generated directly by ZELINK (using the full ZEMAX ray-trace approach) against the sum of the products of the column vector of instantaneous mechanical DOF perturbations and each row from the coefficient sensitivities matrix (yielding each predicted coefficient value), and the corresponding Zernike polynomial basis function, with these products summed over the number of terms kept in the Zernike expansion. These two vectors were reshaped into 2D arrays and plotted next to each other for visual comparison.

To provide quantitative comparison, normalized RMS errors between the OPDs generated by ZELINK and those generated by the linear-sensitivity approach were computed, and are tabulated in Table I. The largest numbers in table 2 correspond to the perturbations that are rotations about the Z-axis, which is an axis of symmetry for this particular optical system. As such, Z-axis rotations have no impact on the OPD and produce extremely small OPD numbers relative to those produced by other DOF motions, dominated by noise from truncations made during computation. Thus, the values in Table I corresponding to the Z-axis rotations are spurious and due to the effects of numerical noise. Overall, reconstruction errors were found to be less than $1 \%$ for all cases not corresponding to $\mathrm{Z}$-axis rotations, for all perturbation magnitudes up to the $\pm 10^{-6} \mathrm{~m}$ or rad limits, at all points within the pixellated pupil plane at which the actual OPD value was not very close to or at zero relative to the mean across the actual OPD data surface. 


\section{B. Diffraction Effects and Image Generation}

Diffraction effects can be simulated through the use of spatial Fourier techniques that are in general very similar to standard temporal Fourier techniques used in dynamic systems analysis. The Fourier techniques employed here for optical propagation, relying on the theory of linear systems, are only valid in the Fraunhofer region and are in general only valid over a limited regime around an operating point. This is because of the breakdown, at large perturbations, of the underlying assumptions made in the derivation of those techniques, such as the linearity of the system, spatial invariance (isoplanicity) of the impulse response (PSF), and absence of effective discontinuities (due to sampling limits) in the data surfaces to be transformed. For a more detailed discussion of the derivations and assumptions made, the reader is referred to [17].

A map of OPD at the pupil plane represents all of the aberrations within the optical system along the propagation path up to the location of that plane. Let this OPD map be denoted as $\phi\left(S_{x}, S_{y}\right)$, the real 2D wavefront aberration function with units of either waves or radians of phase. As shown in [8], the complex coherent transfer function (CTF), denoted $W(\xi, \psi)$, for the system is found as

$$
\begin{aligned}
W(\xi, \psi) & =A\left(S_{x}, S_{y}\right) e^{i \phi\left(S_{x}, S_{y}\right)} \\
& =A\left(S_{x}, S_{y}\right) e^{i \sum_{j=1}^{n} \alpha_{j} Z_{j}\left(S_{x}, S_{y}\right)},
\end{aligned}
$$

where $\alpha_{j}, Z_{j}$ are the $j$ th Zernike coefficient and polynomial shape respectively, and where $A\left(S_{x}, S_{y}\right)$ is the binary pupil extents definition, defined as unity inside the system aperture, and zero elsewhere. Following [8], taking the autocorrelation of $W(\xi, \psi)$ yields the complex optical transfer function (OTF) as

$$
\Pi(\xi, \psi)=\iint W^{*}\left(\sigma_{\xi}, \sigma_{\psi}\right) W\left(\xi-\sigma_{\xi}, \psi-\sigma_{\psi}\right) d \sigma_{\xi} d \sigma_{\psi} .
$$

Given the Fourier transform of an object intensity map, $O(\xi, \psi)$, the corresponding Fourier transform of the image generated of the object, denoted $I(\xi, \psi)$, by the optical system can be computed as

$$
I(\xi, \psi)=\Pi(\xi, \psi) \cdot O(\xi, \psi),
$$

where - denotes element-by-element (Haddamard) multiplication. The corresponding spatial image can then be computed via standard inverse Fourier transform methods.

An object intensity map was arbitrarily defined. Given an arbitrary OPD generated by the process outlined in Section III-A above, a measurable image intensity profile at the image plane was generated for this object using the steps outlined above. To provide the dual images required for phase diversity type algorithms, the same calculations were also performed for a second OPD surface where a small amount of defocus aberration was added, and the nominal and defocused images were set as system outputs.

\section{PHASE DIVERSITY ALGORITHM}

Phase diversity approaches represent a form of opticsbased metrology where perturbations present in the system are determined not by direct measurement, but rather by the effects that they generate in images produced by the system. In particular, phase diversity approaches use the nominal image produced by the system and a second image, which contains a small but known amount of additional aberration (the diversity), to back-calculate the system aberration function (the system OPD) through the minimization of an error metric. Defocus aberrations tend to be the most widely utilized in practice for these techniques, due to their ease of implementation in optical hardware.

The attraction of phase diversity techniques are twofold: first, they rely solely on image-based information, negating the need for complex metrology systems, and second, they are scene-independent; that is, they do not require a particular type of image or scene in order to work, unlike approaches where a point source or other calibrated scene are used to divine system aberrations.

A particular phase diversity technique is based on the so-called Gonsalves metric, given by (see [8],[9]):

$$
E . M .=\sum_{\xi, \psi}\left(\frac{\left|I_{\mathrm{n}}(\xi, \psi) \cdot \Pi_{\mathrm{d}}(\xi, \psi)-I_{\mathrm{d}}(\xi, \psi) \cdot \Pi_{\mathrm{n}}(\xi, \psi)\right|^{2}}{\left|\Pi_{\mathrm{n}}(\xi, \psi)\right|^{2}+\left|\Pi_{\mathrm{d}}(\xi, \psi)\right|^{2}+\alpha}\right),
$$

where $I_{\mathrm{n}}, \Pi_{\mathrm{n}}$ and $I_{\mathrm{d}}, \Pi_{\mathrm{d}}$ denote the image Fourier transform and the OTF of the nominal system and the diversity (defocused) system, respectively, and $\alpha$ is a small regularization constant used to prevent singularity. Note that $\Pi_{n}$ and $\Pi_{d}$ are indirect functions of the unknown Zernike coefficients.

Unfortunately, the function represented by (10) cannot be proven to be convex, and the implementation of phase diversity approaches essentially result in an unconstrained nonlinear optimization, where the elements within the Zernike coefficients vector are the parameters solved for to minimize the error metric above.

Standard optimization routines in MATLAB were used to compute solutions to the optimization problem, with particular attention given to how much the initial parameter guess could deviate from actual parameter values before poor results were encountered, i.e. before very many iterations were required to converge to the actual values. A deviation towards $+\infty$ was found to be more favorable than one towards $-\infty$ in this respect. Like this deviation, the perturbations introduced to the system had to be within a narrow range for convergence to result. As stated within [10], the phase diversity algorithm breaks down if the total phase variation across the pupil plane exceeds one wave. This imposed a restriction on the values of the actual Zernike coefficients to be found, which in turn translated to range limits on the DOF motions of the structure and in turn range limits on the PZT actuator voltages (the perturbations). It was concluded that the phase diversity algorithm converged reliably but not quickly or efficiently 


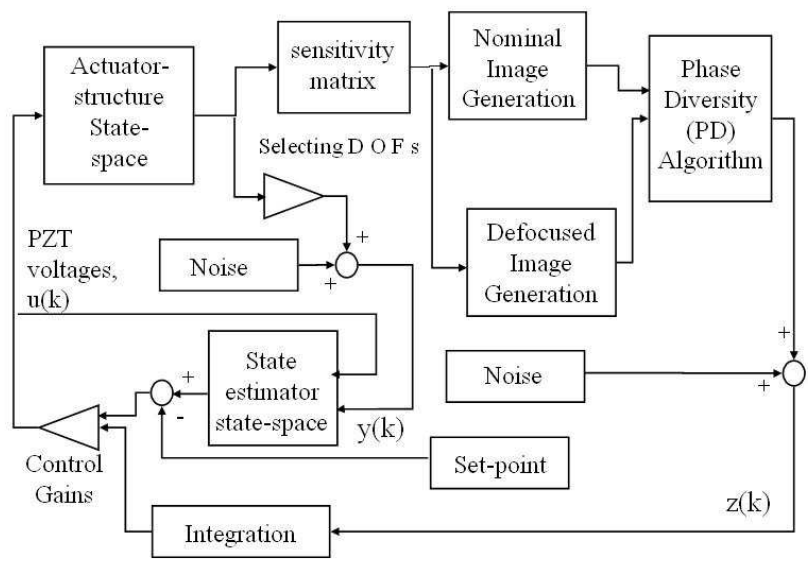

Fig. 4. Overall System Diagram with Feedback Control

enough (requiring up to 500 iterations), to use on-line in the simulations performed to test the control design below, given limited computing resources. Thus a fast standin function employing knowledge of the actual Zernike coefficient values from within the plant in addition to a stochastic component was used to simulate the phase diversity algorithm results for this purpose.

\section{CONTROL SYSTEM ARCHITECTURE}

Fig. 4 shows the diagram for the overall system with the controller added to it. The two sets of signals available from the model of the opto-mechanical system for use with the control system were laser metrology sensor signals, $y(k)$, and the Zernike coefficient signals from the phase diversity algorithm, $z(k)$. The former was modeled via the addition of physically realistic noise to selected mechanical DOF perturbations output by the actuator-structure state-space system, while the latter was modeled with the addition of noise to the Zernike coefficients generated by the linear sensitivity process described in III-A. The key performance vector driven to zero through control was $z(k)$, which served as the true measure of the aberration in the wavefront coming into the observation instrumentation (camera/CCD). An adaptation of the Kalman-Bucy filter was derived in discrete time, with the additional inclusion of biases present in the system due to the non-common optical paths of metrology and observation instrumentation. Extremely low frequency drift due to thermal variation was also considered to be lumped into these biases. Then this control scheme was implemented along with the opto-mechanical model in Simulink.

\section{A. Control Derivation}

Given the discrete time plant (excluding the noise terms) as follows,

$$
\begin{aligned}
x(k+1) & =A x(k)+B u(k) \\
y(k) & =C x(k)+y_{d 1} \\
& =\tilde{y}(k)+y_{d 1},
\end{aligned}
$$

where $y_{d 1}$ represents all non-common path bias and thermal bias on the laser metrology signals $y(k)$. Added to this we have the relationship

$$
z_{\tilde{y}}(k)=\left[\frac{\partial z_{\tilde{y}}}{\partial \tilde{y}}\right] \tilde{y}(k),
$$

where $\left[\partial z_{\tilde{y}} / \partial \tilde{y}\right]$ is the sensitivity matrix obtained above. Multiplying the interferometer output by this sensitivity matrix gives $z_{y}(k)$ as

$$
\begin{aligned}
z_{y}(k) & =\left[\frac{\partial z_{\tilde{y}}}{\partial \tilde{y}}\right] \tilde{y}(k)+\left[\frac{\partial z_{\tilde{y}}}{\partial \tilde{y}}\right] y_{d 1} \\
& =z_{\tilde{y}}+z_{d 1}
\end{aligned}
$$

The phase-diversity output $z(k)$ can be represented (in similar fashion to the interferometer output) as

$$
\begin{aligned}
z(k) & =\left[\frac{\partial z_{\tilde{y}}}{\partial \tilde{y}}\right] C x(k)+z_{d 2} \\
& =z_{\tilde{y}}+z_{d 2} \\
& =z_{y}(k)-\bar{z}
\end{aligned}
$$

where $\bar{z}=z_{d 1}-z_{d 2}$ is the total constant bias term which acts as a "set-point" that $z_{y}(k)$ is being driven to in order to drive $z(k)$ toward zero as $k \rightarrow \infty$. In this way the control problem can be viewed as an LQ tracking problem. In practice, $\bar{z}$ can be approximated from the expected value of collected measurements of the interferometry and phasediversity outputs, which include both sensor and process white noise, as follows

$$
\bar{z}=E\left[\left[\frac{\partial z \tilde{y}}{\partial \tilde{y}}\right] y(k)-z(k)\right]
$$

The Zernike set-point translates to a state set-point with

$$
\bar{z}=\left[\frac{\partial z_{\tilde{y}}}{\partial \tilde{y}}\right] C \bar{x}
$$

It is assumed the discrete time state estimator is constructed as follows

$$
\begin{aligned}
\hat{x}(k+1) & =A \hat{x}(k)+B u(k)+G(y(k)-\hat{y}(k)) \\
\hat{y}(k) & =C \hat{x}(k) \\
\hat{z}(k) & =\left[\frac{\partial z \tilde{y}}{\partial \tilde{y}}\right] \hat{y}(k)
\end{aligned}
$$

Now we define a series of errors and error differences, starting with the errors of the states and estimated states relative to the state set-point:

$$
\begin{aligned}
\xi(k) & =x(k)-\bar{x} \\
\hat{\xi}(k) & =\hat{x}(k)-\bar{x} \\
\Delta \xi(k) & =\xi(k+1)-\xi(k)=x(k+1)-x(k) \\
\Delta \hat{\xi}(k) & =\hat{\xi}(k+1)-\hat{\xi}(k)=\hat{x}(k+1)-\hat{x}(k) \\
e(k) & =\hat{\xi}(k)-\xi(k)=\hat{x}(k)-x(k) \\
\Delta e(k) & =\Delta \hat{\xi}(k)-\Delta \xi(k) \\
& =\hat{x}(k+1)-\hat{x}(k)-x(k+1)+x(k)
\end{aligned}
$$


With these definitions in hand, the plant error propagation system is given by

$$
\begin{aligned}
\xi(k+1) & =A \xi(k)+B u(k)+(A-I) \bar{x} \\
e_{y}(k) & =y(k)+C \bar{x} \\
& =C \xi(k)+y_{d 1}
\end{aligned}
$$

Similarly, the estimator error propagation system is seen to be, with some substitution,

$$
\begin{aligned}
\hat{\xi}(k+1)= & A \hat{\xi}(k)+B u(k)+G C(\xi(k)-\hat{\xi}(k))+\ldots \\
& \ldots(A-I) \bar{x}+G y_{d 1} \\
\hat{e}_{y}(k)= & C \hat{\xi}(k) \\
\hat{z}(k)= & \hat{z}_{y}(k)-\bar{z}=\left[\frac{\partial z_{\tilde{y}}}{\partial \tilde{y}}\right] C \hat{\xi}(k)
\end{aligned}
$$

To eliminate the constant terms from these error propagation systems, we define $\Delta u(k)=u(k+1)-u(k)$ and assume the overall discrete system has a positive sample time of $\Delta t$, then formulate the corresponding propagation systems for the error differences, with division of the equations by the sample time. For the plant this produces

$$
\frac{\Delta \xi(k+1)}{\Delta t}=A \frac{\Delta \xi(k)}{\Delta t}+B \frac{\Delta u(k)}{\Delta t}
$$

And for the estimator

$$
\frac{\Delta \hat{\xi}(k+1)}{\Delta t}=A \frac{\Delta \hat{\xi}(k)}{\Delta t}+B \frac{\Delta u(k)}{\Delta t}+G C\left(\frac{\Delta \xi(k)}{\Delta t}-\frac{\Delta \hat{\xi}(k)}{\Delta t}\right)
$$

Using the definition of $\Delta e(k)$ from above, this estimator error difference can be rewritten as

$$
\frac{\Delta e(k+1)}{\Delta t}=(A-G C) \frac{\Delta e(k)}{\Delta t}
$$

Equations (29) and (31) need to be augmented by a propagation of the key performance vector of $z(k)$ to to ensure that this is driven to zero, before combination into the overall system matrices. For this propagation, we observe that

$$
\begin{aligned}
z(k+1) & =z(k+1)-z(k)+z(k) \\
& =\left[\frac{\partial z_{\tilde{y}}}{\partial \tilde{y}}\right] C(\xi(k+1)-\xi(k))+z(k) \\
z(k+1) & =\Delta t\left[\frac{\partial z_{\tilde{y}}}{\partial \tilde{y}}\right] C \frac{\Delta \xi(k)}{\Delta t}+z(k)
\end{aligned}
$$

Now combining 29, 31 and 32 yields:

$$
\begin{gathered}
{\left[\begin{array}{c}
\frac{\Delta \xi(k+1)}{\Delta t} \\
z(k+1) \\
\frac{\Delta e(k+1)}{\Delta t}
\end{array}\right]=\left[\begin{array}{ccc}
A & 0 & 0 \\
\Delta t\left[\frac{\partial z_{\tilde{y}}}{\partial \tilde{y}}\right] C & I & 0 \\
0 & 0 & (A-G C)
\end{array}\right]\left[\begin{array}{c}
\frac{\Delta \xi(k)}{\Delta t} \\
z(k) \\
\frac{\Delta e(k)}{\Delta t}
\end{array}\right]} \\
\ldots+\left[\begin{array}{c}
B \\
0 \\
0
\end{array}\right] \frac{\Delta u(k)}{\Delta t}
\end{gathered}
$$

Assuming the control law to have the form

$$
\begin{aligned}
\frac{\Delta u(k)}{\Delta t} & =-K_{1} \frac{\Delta \hat{\xi}(k)}{\Delta t}-K_{2} z(k) \\
& =-K_{1}\left(\frac{\Delta \xi(k)}{\Delta t}+\frac{\Delta e(k)}{\Delta t}\right)-K_{2} z(k)
\end{aligned}
$$

yields the following closed-loop system matrix

$$
\left[\begin{array}{ccc}
\left(A-B K_{1}\right) & -B K_{2} & -B K_{1} \\
\Delta t\left[\frac{\partial z_{\tilde{y}}}{\partial \tilde{y}}\right] C & I & 0 \\
0 & 0 & (A-G C)
\end{array}\right],
$$

from which the stability of the entire system is determined. It is still necessary to determine the form of the control law, $u(k)$, and for this we step through (34) given that for all $i<0, \hat{\xi}(i)=0$ and that we have a non-trivial initial error in the state estimate, or $\hat{\xi}(0) \neq 0$. This produces the recursive equation

$$
u(k)=-K_{1} \hat{\xi}(k)-K_{2} \Delta t \sum_{i=0}^{k-1} z(i)
$$

Defining integrator states as $x_{I}(k)=\Delta t \sum_{i=0}^{k-1} z(i)$ and the integrator state-space system as

$$
x_{I}(k+1)=x_{I}(k)+\Delta t z(k)
$$

allows this control law to be rewritten as

$$
u(k)=-K_{1} \hat{x}(k)+K_{1} \bar{x}-K_{2} x_{I}(k)
$$

using the definition of the state estimate error as well. This primary result is the proportional plus integral type of controller expected to compensate for the biases and drive the $z(k)$ representation of the wavefront error to zero.

\section{B. Control Implementation}

In implementing the above within Simulink, six of the 12 DOF outputs from the plant-state space system (Z-piston, $\mathrm{X}$-tilt, and Y-tilt for the primary and secondary mirrors each) were picked out and summed with modeled noise. The stand-in phase diversity function was eventually set to provide only three Zernike coefficients, to which the modeled zero-mean white noise was added. The feedback loop was opened and the system was simulated to record to the workspace a sampling of $y(k)$ and $z(k)$ so that $\bar{z}$ could be computed by (18). Then the feedback loop was closed, with the $K_{b a r}=\left[\begin{array}{ll}-K_{1} & -K_{2}\end{array}\right]$ and the $G$ having been obtained by LQR and LQG solutions respectively. Originally, more Zernike coefficients had been provided by the phase diversity function to the integrator state-space system input, but this resulted in poor scaling for the LQR problem. This was due to the relatively small participation of most all higher order Zernike coefficients in the OPD map. Weighting/scaling the $Q$ and $R$, then balancing the actuator-structure system and reducing its order was tried unsuccessfully prior to elimination of the additional Zernike coefficient signals. 

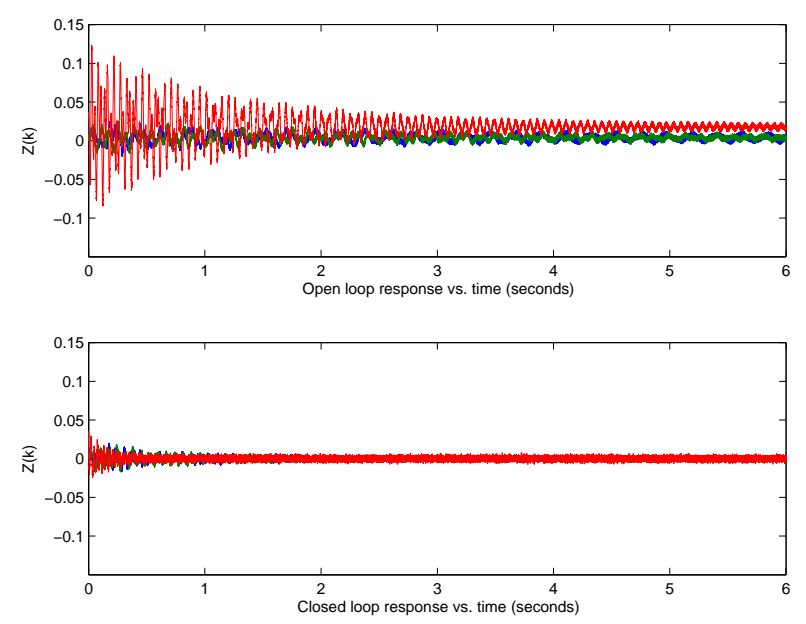

Fig. 5. Time Responses of PD-output Zernike Coefficients, Open and Closed Loop, to Given Initial Condition $x_{0}$

\section{RESULTS}

Introduction of the control system reduced the distance of the eigenvalues from the origin on the S-plane, improving stability. For the original plant state matrix, this distance ranged from $0.999371-0.999950$. For the $(A-G C)$ matrix these values were $0.995262-0.999942$ and for the overall closed loop matrix they were $0.245967-0.999942$. Fig. 5 below shows a comparison of the open loop and closed loop time responses of the Zernike coefficients, output from the simulated phase diversity algorithm, to given initial plant states $x_{0}$, a column-wise vector of $5 \times 10^{-6}$.

\section{CONCLUSIONS AND FUTURE WORK}

The inputs to the structure for the example system are linearly mapped to the coefficients for a particular set of basis functions (the Zernike standard polynomials). These coefficients and basis functions together represent the real OPD at the pupil plane (nearly co-located with the camera/CCD for the system), which is then driven to zero using the linear feedback control. Therefore the actual image distortion is being eliminated in addition to intermediate structural motion. This is significant with respect to new opto-mechanical systems being proposed for future spacecraft missions, in which the combination of large sizes and light-weighted construction calls into question the assumption that the usual sensing and cancellation of structural motion automatically translates to good image quality. Very low frequency motions and drift/bias in the system not detectable with the interferometer metrology are also corrected for with this approach.

Limitations on the usefulness of the entire integrated modeling effort described herein include significant computational overhead associated with use of the phase diversity algorithm on-line, although faster phase diversity algorithms are under development. In addition, the work done to date has not included flexible modes for the mirror surfaces, which is unrealistic given the membrane-like mirrors proposed for many new opto-mechanical systems. Future work may be needed to introduce these flexible modes, requiring increases in the number of DOF channels, the size of the sensitivities matrix, and the number of Zernike coefficients used as parameters in the phase diversity algorithm so as to capture all major OPD behaviors. It is also desirable to extend the approach presented here to multiple-segment sparse-aperture imaging systems for which highly asymmetric OPDs are observed with independent motion of each segment. Derivation of a new orthogonal basis function set from observed OPDs may be needed as an alternative to use of Zernike standard coefficients and functions, so as to capture the OPD behavior with a smaller number of coefficients and thereby reduce the computation required for modeling and operating the system as a whole.

\section{REFERENCES}

[1] Przemieniecki, J. S. Theory of Matrix Structural Analysis. New York: McGraw-Hill, Inc., 1968.

[2] Junkins, J.L. and Kim, Y. Introduction to Dynamics and Control of Flexible Structures. AIAA Education Series. AIAA: Washington, D.C., 1993.

[3] Breault Research Organization. Modeling and Analysis for Controlled Optical Systems User Manual, version 2.0. Breault Research Organization, 1996.

[4] JPL Publication D-9816. Controlled Optics Modeling Package User Manual, release 1.0. Pasadena, CA: National Aeronautics and Space Administration, Jet Propulsion Laboratory, California Institute of Technology, 1992.

[5] JPL Publication 98-12. Integrated Modeling of Optical Systems User's Manual, release 4.0. Pasadena, CA: National Aeronautics and Space Administration, Jet Propulsion Laboratory, California Institute of Technology, 1998.

[6] Craig, L., et al., "Integrated Modeling of a Semigrigid Hybrid Mirror and a Highly Actuated Membrane Mirror as Candidates for the Next Generation Space Telescope," Proc. SPIE, vol. 4013, pp. 810-825, 2000.

[7] de Weck, O., Miller, D., Mallory, G., Mosier, G., "Integrated Modeling and Dynamics Simulation for the Next Generation Space Telescope (NGST)," Proc. SPIE, vol. 4013, pp. 920-934, 2000.

[8] Gonsalves, R. A. and Chidlaw, R., "Wavefront Sensing by Phase Retreival," Proc. SPIE, vol. 207, pp. 32-39, 1979.

[9] Paxman, R. G. and Fienup, R. J., ”Optical Misalignment Sensing and Image Reconstruction Using Phase Diversity," J. Optical Soc. Am. A, vol. 5, pp. 914-923, 1988.

[10] Gavel, D., Bauman, B., Campbell, E., Carrano, C., and Olivier, S., "A Practical Comparison of Phase Diversity to Interferometry in Measuring the Aberrations in an Adaptive Optics System," Proc. SPIE, vol. 3762, pp. 266-268, 1999.

[11] Physik Instrumente GmbH \& Co., "Tutorial: Piezoelectrics in Positioning Contents," http://www.physikinstrumente.de/products/ section4/index.htm, 2/23/2004.

[12] Gaskill, J. Linear Systems, Fourier Transforms, and Optics. New York: J. Wiley and Sons, Inc., 1978.

[13] Wilson, R. G. Fourier Series and Optical Transform Techniques in Contemporary Optics: An Introduction. New York: J. Wiley and Sons, Inc., 1995.

[14] Redding, D., and Breckenridge, W., "Optical Modeling for Dynamics and Control Analysis," Journal of Guidance, Control, and Dynamics, vol. 14, no. 5, pp. 1021-1032, 1991.

[15] Redding, D., Milman, M. and Loboda, G., "Linear Analysis of OptoMechanical Systems," Proc. SPIE, vol. 1696, pp. 84-99, 1992.

[16] Focus Software, Inc. ZEMAX Optical Design Program User's Guide, version 9.0. Tucson: Focus Software, Inc., 2000.

[17] Goodman, J. W. Introduction to Fourier Optics. New York: McGrawHill, Inc., 1968. 